\title{
Ueber 455 Fälle von Eclampsie aus der St. Petersburger Gebairanstalt.
}

Von

\section{E. Bidder.}

Die Discussion über Eclampsie ist augenblicklich wieder in den Vordergrund des Interesses getreten und erstreckt sich auf alle Seiten der Frage, die theoretischen sowohl als die practischen. Neue Perspectiven eröffnen sich auf neue Anschaungen über Pathogenese, vielleicht auch Therapie, der genannten gefürchteten Krankheit. Vor Zeiten habe ich selbst ${ }^{7}$ ), freilich in ganz anderer Richtung, als es jetzt geschieht, experimentell in diesem Gebiete gearbeitet; es lag mir daher nahe, das grosse Material, das ich seither unter den Händen gehabt, zu einem Ueberblick dessen zu benutzen, was wir bisher dem schlimmen Feinde gegenüber haben leisten können. So rückschauend werde ich mir über die Theorie und Pathogenese der Eclampsie auch nur wenige Worte gelegentlich erlauben; darüber zu sprechen, halte ich im Augenblick für ungeeignet, da voraussichtlich an vielen Orten - auch bei uns in dieser Richtung gearbeitet wird. Meine Angaben werden im Wesentlichen statistische und klinische sein. Olshausen ${ }^{2}$, Löhlein $^{3}$ ), Dührssen ${ }^{4}$ ) und Andere haben in jüngster Zeit ältere und jüngere Statistiken mit der ihrigen verglichen; ich kann mich

1) Bidder. Experimentelle Beiträge zur Eclampsiefrage in Beiträge z. Gynäk. u. Geburtskunde v. Holst. II. Hft. Tübingen 1867.

2) 0lshausen. Ueber Eelampsie. Volkm, klin. Vortr. N. F. 39.

3) Löhloin. Ueber Häufigheit, Prognose und Behandlung der puerperalen Eclampsie. Gynäk. Tagesfragen. Hft. 2.

4) Dührssen. Ueber Eclampsie. Dieses Archiv Bd. 42, 43. 
daher in dieser Beziehung auf das Nothwendigste beschränken und auf die genannten schönen Arbeiten verweisen.

Mein Material besteht aus 455 Fällen, die vom 1. Januar 1873 bis zum 31. December 1891 im St. Petersburger Gebärhanse beobachtet worden sind. In demselben Zeitraum sind 60583 Frauen im Hause verpflegt worden, es kommt also 1 Fall von Eclampsie auf 133. Dabei muss aber bemerkt werden, dass die Häufigkeit der Eclampsie bei uns stetig zunimmt: In den Jahren 1873-1880 betrug sie $1: 151,1881-1891$ dagegen $1: 123$, und in den letzten 4 Jahren 1888-1891 inel. sogar 1:100, eine Zahl, die nur von wenigen anderen Anstalten übertroffen wird. Diese zunehmende Häufigkeit ist ein Factum, das in einzelnen anderen Anstalten ebenfalls beobachtet worden ist, z. B. in dem Moskaner Gebärhaus ${ }^{1}$ ) und der Berliner Charité $^{2}$ ). Daraus zu folgern, dass die Eclampsie überhaupt jetzt häufiger auftritt als früher, wäre natürlich verfehlt, zum Wenigsten verfrüht. Die Thatsache lässt sich auch erklären durch das wachsende Vertrauen der Bevölkerung zu einer Anstalt. Bei uns spricht letzteres sich aus in dem Factum, dass die Frequenz der Anstalt sich in den besprochenen Jahren auf das Doppelte vermehrt hat, während die Bevölkerung Petersburgs nur unbedeutend gewachsen ist. Ziehen wir, um einen etwas präciseren Begriff von der Häufigkeit der Eclampsie zu bekommen, mit Löhlein nur die autochthonen Fälle in Betracht, d. h. diejenigen, in welchen die Eclampsie erst nach der Aufnahme in die Anstalt ausbrach, so erhalten wir folgende Zahlen (hier nur für die Jahre 1881-1891): Auf 41177 Geburten 327 Fälle von Eclampsie, davon 87 autochthon, d. h. 1:473. Hiernach würden wir in der Mitte stehen zwischen der von Löhlein durch seine Sammelforschung gefundene ZahI 1:330 und den grossen Wiener Kliniken, die $1: 589$ angeben. Hieraus lässt sich nun aber schwerlich der Schluss ziehen, dass verschiedene Berölkerungen für Eclampsie verschieden disponirt seien. Gar zu viele andere Momente kämen auch noch in Betracht. Sollte hier z. B. die mehr oder weniger intensive Ausnutzung des Materials als Untersuchungsund Lehrobject nicht vielleicht eine Rolle spielen? Abgesehen von allen Theorien über die Pathogenese der Eclampsie steht doch

1) Pawpertow. Arbeiten des 4. Congresses russischer Aerzte. Moskau 1892 (russisch).

2) Löhlein a. a. 0. pg. 84. 
als Thatsache fest, dass der eclamptische Anfall ausgelöst wird von einer ganzen Reihe äusserer Reize, zu denen auch eine Untersuchung gehört.

Was die Beziehung der Eclampsie zur Jahreszeit betrifft, so bekommen wir bei Zusammenstellung nach Monaten Schwankungen, deren Unregelmässigkeit an sich schon beweist, dass unsere Zahlen für eine derartige Untersuchung offenbar zu klein sind. Nach Jahreszeiten geordnet, bekommen wir für März bis Mai 130, Juni bis August 107, September bis November 97, December bis Februar 111. - Pawpertow (a. a. 0., pag. 886) giebt an für den Frühling 69, Sommer 78, Herbst 54, Winter 87. Zufällig stimmen unsere Zahlen darin überein, dass der Herbst am wenigsten belastet ist. Alles andere stimmt nicht.

Das gruppenweise Auftreten der Eclampsie, wie es Olshausen (a. a. 0. pag. 326) wieder einmal hervorbebt und sogar durch Zahlen zu beweisen sucht, lässt sich in dieser Weise ja wohl constatiren. Es scheint mir aber, dass diese Thatsache sich kaum über den Bereich des "Zufalls" erhebt, über das Gebiet des sogenannten „Gesetzes der Duplicität der seltenen Fälle“ und kann jedenfalls nicht für die Theorie der Eclampsie verwerthet werden, was doch der Zweck solcher Angaben ist. Den Eindruck von epidemischem Auftreten der Eclàmpsie habe ich nie bekommen, ebensowenig den von Uebertragbarkeit derselben. Aufzeichnungen über Wohnort, Strasse, Hausnummer, aus denen die betreffenden Patientinnen kamen, wärden in Bezug auf die genannten Fragen vielleicht wichtiger sein. Das ist noch nicht versucht worden.

Das Ueberwiegen der Erstgebärenden muss ich natürlich ebenso constatiren, wie alle anderen Autoren. Ich zähle 337 Primiparae $=74,3$ pCt. und 116 Multiparae (in 2 Fällen fehlt die Notiz), also genau die Zahlen von Olshausen und Löhlein. Auf die Gesammtzahl der Frauen bezogen, ergiebt das eine Eclampsie auf 53 Erstgebärende und 368 Mehrgebärende; bei ersteren ist die Eclampsie also $7 \mathrm{Mal}$ häufiger als bei letzteren.

Ueber das Alter der Kranken gebe ich folgende kleine Tabelle:

$\begin{array}{lccr} & \text { Primiparae } & \text { Multiparae } & \text { Summa } \\ \text { 16-19 Jahre } & 86 & \mathbf{1} & 87 \\ 20-24 & 148 & 34 & 182 \\ 25-29 " & 64 & 35 & 99 \\ 30-34 \quad " & 24 & 18 & 42\end{array}$




$\begin{array}{lccc} & \text { Primiparae } & \text { Multiparae } & \text { Summa } \\ \text { 35-39 Jahre } & 8 & 20 & 28 \\ \text { 40 Jahre und mehr } & 3 & 6 & 9 \\ \text { ? Jahre } & 4 & 2 & 6\end{array}$

Ich glaube nicht, dass sich aus diesen Zahlen eine besondere Bevorzugung irgend einer Altersstufe erschliessen lässt; bei den Mehrgebärenden sind ausserdem die Zahlen entschieden zu klein. Unsere 35 Erstgebärende mit einem Alter von über 30 Jahren geben 10 pCt. der eclamptischen Primiparae, während Steinmann ${ }^{1}$ ) für alte Erstgebärende an unserer Anstalt überhaupt eine Frequenz von 7,1 pCt. berechnet hat. Trotz der bekannten Disposition alter Erstgebärender für Nierenkrankheiten, die Winckel in seinen Dresdener Berichten so prägnant hervorgehoben, ist ihre Disposition für Eclampsie doch nur wenig höher, als die allgemeine. Dies bestätigt die altbekannte Thatsache, dass Schwangere, die an chronischen Nierenkrankheiten leiden, sehr häufig von Eclampsie verschont bleiben. Zum zu Stande kommen der Eclampsie gehört jedenfalls noch eine besondere Noxe, die nicht mit der Nierenkrankheit gegeben ist.

Von den Mehrgebärenden kamen zum 2.-4. Nal nieder 93. zum 5. Mal und mehr 23, darunter 4 zum 10., 2 zum 11, 1 zum 14. Male. Die Vielgebärenden sind bei uns also auch nicht so stark vertreten, wie bei Olshausen.

Mehrfrüchtige Geburten zählen wir 34, und zwar 33 Zwillings- und 1 Drillingsgeburten, d. h. unter unseren Eelamptischen haben wir „mehrfache“ Geburten im Verhältniss von 1:13 oder 7,4 pCt., während wir im Allgemeinen 1:46 oder 2,1 pCt. mehrfache Geburten zählen ${ }^{2}$ ). Das sind Zahlen, die denen von Schauta ${ }^{3}$ ) am nächsten stehen. Sie beweisen, dass mehrfache Schwangerschaft mehr zur Eclampsie disponirt, als einfache.

Frühgeburten. Unter unseren 455 Fällen ist die Eclampsie eingetreten im 5. Monat der Schwangerschaft 1 Mal, im 6. $1 \mathrm{Mal}$, im 7. $7 \mathrm{Mal}$, im 8. $21 \mathrm{Mal}$, im 9. $49 \mathrm{Mal}$, im 10.61 Mal, in Summa bei 140 vorzeitigen und 313 rechtzeitigen Geburten (in 2 Fällen fehlt die Notiz). 30 pCt. sämmtlicher Eclampsieen fallen also auf Frühgeburten. Beziehen wir diese Zahlen auf die Gesammt-

1) Steinmann. Dieses Archiv Bd. XII.

2) Bidder and Sutugin, Bericht.

3) Schauta. Dieses Archir Bd. XVIII. 
zahl aller bei uns beobachteten rechtzeitigen Geburten und der frühzeitigen rom 5.-10. Monat, so kommt bei ersteren 1 Eclampsie auf 179 , bei letzteren auf 31 Geburten. Vor dem 5. Schwangerschaftsmonat haben wir keine Eclampsie gesehen. Aus den obigen Zahlen können wir also schliessen, dass in den Schwangerschaftsmonaten vom 5.-10. die Eclampsie verhältnissmässig wenigstens 5 Mal so häufig auftritt, als am Ende der Schwangerschaft.

Der Maassstab, nach dem ich Frühgeburten messe, ist aber ein etwas anderer, als 0lshausen ihn anwendet. Er betrachtet, wenn Zeitangaben fehlen, als frühgeboren alle Kinder, die mit einem Gewicht von weniger als $2250 \mathrm{~g}$ (bei Zwillingen $2000 \mathrm{~g}$ ) zur Welt kommen. Diese untere Gewichtsgrenze scheint mir zu niedrig angesetzt zu sein. Von meinen 278 Einzelkindern (Zwillinge ausgeschlossen), bei denen die Zahlen brauchbar sind, haben nur 57 ein Gewicht unter $3000 \mathrm{~g}$ gehabt, und von diesen nur 4 ein Gewicht unter $2700 \mathrm{~g}$, nämlich 3 Kinder $2600 \mathrm{~g}$ und $12580 \mathrm{~g}$. Unter $2500 \mathrm{~g}$ habe ich keines als ausgetragen angenommen, und zähle daher 30 pCt., ebensoviel wie 0lshansen, würde aber, nach seinem Maassstabe gemessen, viel weniger haben müssen.

Aus der Häufigkeit der Eclampsie bei Frühgeburten zieht Olshausen den Schluss auf ,sehr häufigen Beginn der Erkrankung in der Schwangerschaft mit bald beginnender Geburtsthätigkeit." Dieser Schluss, dass die Eclampsie sehr häufig vor Beginn der Wehen einsetzt, und dass die Wehen erst durch die Eclampsie ausgelöst werden, kann so ohne Weiteres doch nicht zugegeben werden. Beispielsweise haben wir unter unseren Frühgeburten 27 pCt., bei denen die Eclampsie erst in der Nachgeburtsperiode resp. post partum aufgetreten ist. Unser ganzes Eclampsiematerial giebt 29 pCt. solcher Spät-Eclampsieen. Die Frühgeburten haben also, was die Erregang der Wehenthätigkeit durch die Eclampsie betrifft, Nichts vor den rechtzeitigen Geburten voraus, sonst müssten sie bei ihnen häufiger, als bei rechtzeitigem Eintritt der Eclampsie in der ersten Geburtszeit, oder ante partum beobachtet werden. Auf die gegenseitige Abhängigkeit von Eclampsie und Wehenthätigkeit komme ich gleich näher zu sprechen.

Ordnen wir unser Material nach dem Zeitpunkt des Eintritts der Eclampsie, so erhalten wir folgende Tabelle: 
Der erste eclamptische Anfall trat ein 1):

\begin{tabular}{|c|c|c|c|}
\hline \multicolumn{2}{|c|}{ bei Erstgeb. } & bei Mehrgeb. & Summa \\
\hline ante partum & 29 & 9 & 38 \\
\hline in per. I & 78 & 26 & 104 \\
\hline$n \quad, \quad$ II & 84 & 22 & 106 \\
\hline$n \quad "$ III & 35 & 11 & 46 \\
\hline$\because \quad$ IV & 23 & 2 & 25 \\
\hline$n \quad " \quad \mathrm{~V}$ & 7 & 4 & 11 \\
\hline post partum & 81 & 42 & 123 \\
\hline & 337 & 116 & 453 \\
\hline
\end{tabular}

(In 2 Fällen ist die Zeit des Eintritts des ersten Anfalls nicht notirt.) Es versteht sich von selbst, dass erste und zweite Periode nicht streng von einander geschieden werden konnten, ebenso wenig, z. B. bei vorzeitigem Blasensprung, die 2. und 3. Periode: endlich liess sich in manchen Fällen, die in die erste Periode gezählt sind, nicht bestimmen, was zuerst eingetreten war, der erste Anfall oder die erste Wehe.

Aus der Tabelle ist ersichtlich, dass wenigstens in der Hälfte der Fälle der Ausbruch der Eclampsie zusammenfällt mit der Zeit, wo die Wehenthätigkeit im Gange ist. Sehr bevorzugt ist dabei die Eröffnungsperiode, so dass der Einfluss der Wehe sicher nicht von der Hand zu weisen ist. Wenn wir aber daneben sehen, dass in nicht weniger als 172 Fällen die Eclampsie eintrat zu einer Zeit, wo der Uterus nicht arbeitete, und zwar in 38 Fällen vor Beginn der Wehenthätigkeit und in 134 Fällen in der Nachgeburtszeit und post partum, d. h. mehr oder weniger spät nach Vollendung der Geburt des Kindes, - so werden wir unwillkürlich dazu gedrängt, der Wehenthätigkeit keinen so grossen Einfluss auf das Zustandekommen der Eclampsie zuzuschreiben, als es vielfach geschieht. Wir müssten denn das Travail insensible vor dem eigentlichen Beginn der Geburt und die Nachwehen den richtigen Geburtswehen gleichwerthig setzen.

Wie wenig unter Umständen die Wehen nöthig sind, beweist ein Fall von Sutugin ${ }^{2}$ ), der am ersten Tage nach einem Kaiserschnitte nach Porro 2\% resp. 5 Stunden nach der Operation eclamptische Anfälle beobachtete. Eine Reihe interessanter Einzelheiten entdeckten wir bei näherer Betrachtung der Fälle, in denen

1) Wir theilen hier die Geburt in 5 Perioden, weil die Zeit dadurch besser präcisirt wird.

2) 55 Laparotomien, in Moskau ausgeführt. St.Petersburg 1891 (russisch). 
ganz ohne Zweifel die Eclampsie vor Beginn der Wehen einsetzte. Solcher Fälle zähle ich 34 ; in ihnen trat Eclampsie auf von 16 Tagen bis zu wenigen Stunden vor Anfang der Wehen. In 24 von diesen Fällen hörte die Eclampsie auch wieder vor Beginn der Geburt auf und letztere, ebenso wie das Wochenbett, verliefen ohne Anfälle - eine schon oft beobachtete Thatsache. Zwei Schwangere aus dem 8. resp. 10. Schwangersehaftsmonat wurden nach überstandener Eclampsie gesund entlassen und weitere Nachrichten über sie fehlen. In zwei källen starben die Patientinnen, ohne dass sich Wehen eingestellt hätten ( 1 Mal Sectio caesarea in mortua - todtes Kind; das andere Mal wurde der Kaiserschnitt unterlassen, weil die Schwangerschaft nur bis zum 7. Monate vorgeschritten war). In 2 anderen Fällen wurde in höchster Lebensgefahr der Kaiserschnitt ausgeführt, was später näher erörtert werden soll. Die letzten 4 Fälle zeichnen sich dadurch aus, dass bei der einen Patientin nach einem einzigen, zwei Tage vor der Geburt erfolgten Anfall, der Sopor bis über die übrigens normal verlaufende Geburt fortdauerte, die geborenen Zwillinge und die Mutter aber doch gesund entlassen werden konnten. Bei der zweiten Kranken hörten die 11 Tage ante partum im 7. Sohwangerschaftsmonate eingetretenen Anfälle ( 2 an der Zahl) auf, wegen fortdauernder urämischer Erscheinungen wurde dann die künstliche Frühgeburt eingeleitet, was sehr leicht gelang, und ein $1400 \mathrm{~g}$ schweres Kind todt geboren; die Mutter machte ein normales Wochenbett durch und wurde mit ihrer chronischen, interstitiellen Nephritis entlassen. Im dritten Falle erschienen einige Stunden nach Beginn der Eclampsie Wehen; die Eclampsie danerte fort; nach 22 Anfällen wurde die äussere Wendung auf den Steiss gemacht und bei wenig geöffnetem Muttermunde das Kind am Fuss extrahirt; dasselbe gehörte dem Anfange des 10. Monates an und zeigte beginnende Maceration; die Mutter wurde gesund entlassen. - Im vierten Falle endlich beobachteten wir vor der Geburt zwei Anfälle, während der Geburt drei; die Anfälle hörten auf und nach genügender Erweiterung des Muttermundes wurde das Kind mit der Zange asphyctisch extrahirt, konnte aber nicht wieder belebt werden; bei der Mutter aber dauerte das Coma fort und 54 Stunden post partum starb die Yutter unter zunehmendem Collaps, ohne irgend ein Zeichen puerperaler Erkrankung, was die Section bestätigte.

Wir kommen durch alle diese Beobachtungen zum Schluss, 
dass Contraetionen des Uterus, wie sie die Geburt begleiten, keineswegs eine absolute Vorbedingung für den Eintritt der Eclampsie sind.

Dazu kommt eine Reihe von Fällen, in denen die Eclampsie vor Ende der Geburt, die schon mehr oder weniger vorgeschritten war, trotz Fortdauer der Wehen doch aufhörte, worauf dann die Geburt ihren Fortgang nahm and endlich spontan oder durch Kunsthilfe zu Ende gebracht wurde. In mehreren solcher Fälle kamen die Kreissenden, die schon soporös geworden waren, noch vor Beendigung der Geburt wieder zum Bewusstsein. Eine Wiederkehr der Anfälle nach der Geburt ist in solchen Fällen nur höchst selten beobachtet worden. - Ein Theil der hier gemeinten Fälle zählt auch in der vorigen Gruppe, den Eclampsien ante partum; zusammen haben wir $41, \mathrm{~d}$. h. mit anderen Worten: in $12,3 \mathrm{pCt}$. aller derjenigen Eclampsien, die vor oder während der eigentlichen Geburt einsetzten, hat die Eclampsie schon vor Vollendung der Geburt wieder aufgehört.

Zählen wir dazu noch 134 Fälle, in denen die Eclampsie erst in der Nachgeburtsperiode oder ganz post partum auftrat, so giebt das circa 38 pCt. sämmtlicher Eclampsien, die von den eigentlichen Geburtswehen unabhängig sind. Ich glaube, dass dieser Umstand bedeutend genug ist, um bei Formulirung von Hypothesen über die Pathogenese der Eclampsie einigermassen in's Gewicht zu fallen.

Auf der anderen Seite haben wir aber eine ganze Reihe von Fällen, in denen die Eclampsie mit der Ausstossung des Kindes wie abgeschnitten endigte. Solcher Fälle zählen wir 108 , d. h. 33 pCt. sämmtlicher Fälle von Eclampsie in partu. Dazu kommt eine weitere Reihe ron Fällen, in denen nach der Geburt des Kindes nur noch ein oder ein Päar leichte Anfälle auftraten, in denen also der günstige Einfluss der Entleerung des Uterus sich ebenfalls auffällig erwies. Die Zahl dieser Fälle ist vielleicht kaum geringer, als die der ersten Reihe ${ }^{1}$. . Das ist ein Umstand, der auf unser Handeln einen grossen Einfluss haben muss. Denn wenn wir der Wahrscheinlichkeit von $1: 2$ (vielleicht gar umgekehrt von $2: 1$ ) gegenüberstehen, dass mit Beendigung der Geburt die Anfälle aufhören, so werden wir natürlich die Ge-

1) Ich führe keine Zahlen an, weil die Grenze nicht zu bestimmen ist, jedenfalls nicht nach der Zahl der Anfälle post partum, sondern nach ihrem Character. 
burt zu beendigen suchen, sobald es geschehen kann, ohne die Gefahr für Mutter und Kind wesentlich zu steigern. Darauf komme ich noch zurück.

Noch ein Wort über das Vorkommen macerirter Früchte, da eine neuere Hypothese die Eclampsie zurückführt auf Vergiftung durch Producte des Stoffwechsels der Frucht. In unseren Protokollen sind 13 Früchte als macerirt bezeichnet, von denen aber 8 gestrichen werden müssen, da sie nicht macerirt waren, sondern faultodt, gestorben in Folge der Eclampsie am Anfange lange dauernder Geburten.

In 3 Fällen echter Maceration waren die Kinder ebenfalls in Folge der Eclampsie selbst gestorben, aber mehr oder weniger lange Zeit vor Beginn der Geburt. Ein 4. macerirtes Kind aus dem 6. Schwangerschaftsmonat scheint allerdings vor Beginn der Eclampsie gestorben zu sein, da die Bewegungen desselben 8 Tage vor der Geburt aufhörten, die drei beobachteten Anfälle von Eclampsie aber einen Tag vor Beginn der Geburt eintreten.

Es bleibt also nur ein einziger Fall übrig, in welchem $2 \frac{1}{2}$ Stunden nach der Geburt einer 8 monatlichen macerirten Frucht eine heftige puerperale Eclampsie ausbrauch, die aber mit Genesung endigte. Alle übrigen Kinder waren lebend oder frisch abgestorben, d. h. zur Zeit des Eintritts der Eclampsie lebend gewesen.

Wenn wir damit unsere allgemeinen Zahlen vergleichen, die uns überhaupt $2 \mathrm{pCt}$. macerirte Kinder aufweisen, so müssen wir allerdings aussprechen, dass die Thatsache festzustehen scheint, dass Eclampsie nur äusserst selten bei todtem Kinde eintritt.

Fassen wir zusammen, was wir durch das bisher Gesagte für die Pathogenese der Eclampsie gewonnen haben, so ist es etwa Folgendes:

Die Häufigkeit der Eclampsie scheint in den letzten Jahren in stetem Steigen begriffen zo sein.

Die Eclampsie ist von den Jahreszeiten unabhängig. Epidemisch tritt sie nicht auf; auf Ansteckung weist keine Thatsache hin.

Erste und Zwillingsschwangerschaften sind erheblich bevorzugt, in geringem Grade alte Erstgebärende.

Sehr häufig wird Eclampsie bei Frühgeburten beobachtet; vielleicht beruhen somit beide auf derselben Ursache. 
Die Eclampsie hängt nicht von den Geburtswehen ab, tritt sehr häufig auch ohne solche ein.

Die Ausstossung des Kindskörpers übt in wenigstens der Hälfte der Fälle einen äusserst günstigen Einfluss aus auf den Verlauf der Eclampsie.

Auffallend selten sind bei Eclampsie macerirte Früchte; solche kommen eigentlich nur vor als Folge von in der Schwangerschaft überstandener Eclampsie.

In etwa einem Drittel sämmtlicher Fälle tritt Eclampsie ein zu einer Zeit, wo Stoffwechselproducte des Kindskörpers als Ursache kaum mehr in Frage kommen.

Bei alledem dürfen wir nicht vergessen, dass die Eclampsie nur Schwangere, Kreissende und Wöchnerinnen befällt; die Ursache also, die Eclampsie hervorruft, setzt als Bedingung voraus die Veränderungen, denen der weibliche Körper während des Fortpflanzungsgeschäfts unterworfen ist. So wahrscheinlich es auch ist, dass Eclampsie durch Vergiftung hervorgerufen wird, - bis jetzt ist weder das Gift bekannt, noch auch die Wege, auf denen es zur Wirkung kommt, und jede Hypothese bleibt unvollkommen, die die letzte, oben genannte Bedingung bei Seite lässt.

Die allgemeine Prognose der Eclampsie ist bei uns ganz ausserordentlich günstig im Vergleich mit anderen Angaben: Von den 455 Frauen, die an Eclampsie erkrankten, sind im Ganzen 79 gestorben, das macht 17,3 pCt. Hiervon sind aber 31 an complicirenden Krankheiten gestorben, und zwar an puerperaler Sepsis 27, an croupöser Pneumonie 2, an Recurrens 1, an Urämie in Folge beiderseitiger Nierenschrumpfung 1, - so dass nur 48 der Eclampsie selbst erlegen sind, d. h. 10,5 pCt. Dass wir hierbei nicht willkürlich rechnen, beweisen wir durch Theilung unserer Beobachtangszeit in zwoi Perioden 1873-81 und 1882-91, d. h. vor - und nachantiseptische Periode. In ersterer haben wir 32 Todesfälle $=22,2$ pCt., darunter 13 an Eclampsie $=9,1$ pCt., in letzterer 47 Todesfälle $=15,0$ pCt, davon 35 an Eclampsie $=11,1 \mathrm{pCt}$. In den letzten 5 Jahren haben wir auf $19943 \mathrm{Ge}$ burten 190 Fälle von Eclampsie, d. h. 1:104, davon sind gestorben $24=12,6$ pCt., an Eclampsie $20=10,5$ pCt. und nur 4 an Sepsis. - Löhlein's entsprechende Zahlen sind 23,7 pCt. und 19,38 pCt., und sie bezeichnet er schon als sehr erfreulich. Sie werden schon übertroffen von der Moskauer Entbindungsanstalt (cf. Pawpertow a. a. 0.), die nur 20,8 und 14,5 pCt. angiebt. 
Wir können also behaupten, dass wir die bisher niedrigste Sterblichkeit haben (ausgenommen Winckel, der auf die freilich weit kleinere Zahl von 92 nur 7 Todesfälle hatte).

Specialisiren wir unsere Zahlen, so haben eclamptische Erstgebärende eine Mortalität von 10,9 pCt., Mehrgebärende von 8,6 pCt. Dem Alter der Mütter nach geordnet ergiebt sich folgende Reihe: 16-19 Jahre 11,2 pCt., 20--24 Jahre 9,9 pCt, 25-29 Jahre 11,3 pCt., 30-34 Jahre 9,7 pCt., 35-39 Jahre 3,4 pCt., 40 und mehr Jahre 11,1 pCt., wobei die letzteren Gruppen zu klein sind, um mitgezählt zu werden. Es ergiebt sich wohl ans diesen Zahlen, dass Zahl der Geburt und Alter kaum einen Einfluss auf die Intensität der Erkrankung haben.

Ebensowenig können wir aus Vergleichung der Mortalität nach Frühgeburten und Aborten einerseits, $=12,1$ pCt., und der nach rechtzeitigen Geburten andererseits $=9,5$ pCt., irgend sichere Schlüsse ziehen. Höchstens könnte betont werden, dass die günstigeren Zahlen auf die Geburten fallen, die wahrscheinlich kürzere Dauer hatten, also Mehrgebärende gegen Erstgebärende, rechtzeitige gegen frühzeitige Geburten. Das würde einigermaassen für die Voraussetzung sprechen, die das Placentargebiet als Ausgangspunkt der Vergiftung ansieht.

Anders steht es aber mit dem Einfluss der Geburtsperiode, in der die eclamptischen Anfälle einsetzen. Hier erhalten wir, mit Ausschluss zweier Fälle, in denen die nöthigen Notizen fehlen, folgende kleine Reihe:

$\begin{array}{lccr}\text { Beginn d. Anfäle } & \text { Zahl d. Fälle } & \text { Gestorb. an Ecl. } & \text { pCt. } \\ \text { ante partum } & 38 & 5 & 13,1 \\ \text { in per. I et II } & 210 & 31 & 14,7 \\ \text { " " III et IV } & 71 & 4 & 5,6 \\ \text { " } \text { " V et post p. } 134 & 7 & 5,2\end{array}$

Hieraus sehen wir deutlich, dass die Gefahr der Eclampsie mit der längeren Dauer der Wehenthätigkeit schnell steigt. Zum Theil ist diese Gefahr von der Zahl der Anfälle abhängig, die in den ersten beiden Rubriken unstreitig bei weitem grösser ist, als in den beiden letzten.

Was die Zahl der Anfälle für eine enorme prognostische Bedeutung hat, ergiebt sich aus folgender Zusammenstellung.

In den Jahren 1881-1891 zählen wir 76 Fälle von Eclampsie, in denen 16 und mehr Anfälle beobachtet oder mit Gewissheit 
vorausgesetzt werden konnten; von diesen 76 . Müttern sind 33 gestorben $=43,4$ pCt., und zwar direct an der Eclampsie $24=$ $31,5 \mathrm{pCt}$., wobei zu berücksichtigen ist, dass von den 9 subtrahirten Fällen 5 an Pneumonie starben, wahrscheinlich also wohl auch noch auf Rechnung der Eclampsie geschrieben werden müssen. Ziehen wir die Zahlen von unseren allgemeinen in denselben Jahren gewonnenen Zahlen $a b$, so bleiben 251 Fälle nach mit weniger als 16 Anfällen; unter ihnen haben wir 19 Todte $=7,5 \mathrm{pCt}$. und 14 an Eclampsie gestorbene $=5,5$ pCt., ein ganz gewaltiger Unterschied! Natürlich will ich hiermit nicht behaupten, dass die Zahl der Anfälle in directem Causalitätsverhältniss zur Gefahr steht, denn beide können in paralleler Weise durch die Intensität der Vergiftung bedingt sein. Diese ist aber nicht immer ganz gleichartig. Jedenfalls wissen wir, dass es einerseits Fälle giebt, in denen nur wenige Anfälle zum Tode führen, andererseits solche, in denen trotz einer grossen Zahl von Anfällen die Mütter, und zuweilen sogar die Kinder mit dem Leben davonkommen. Dieser letzteren, in denen Mutter und Kind am Leben blieben, haben wir eine ganze Reihe mit 16-33 Anfällen in Summa, und 16-24 Anfällen während der Geburt, d. h. die nach der Geburt des Kindes erfolgten Anfälle nicht mitgezählt. Das widerspricht der gewöhnlichen Beobachtung, dass das Kind meistentheils schon nach wenigen Anfällen abstirbt. Es ist möglich, dass die Noxe, die die Eclampsie hervorruft, bei dazu geeigneten Individuen leichter Krämpfe auslöst, als bei Andern, so dass die Zahl der Anfälle also nicht nur von der Intensität der Giftwirkung, sondern auch von einer gewissen Prädisposition abhängen dürfte. Damit will ich mich aber keineswegs Herff anschliessen, der das Wesen der Elampsie in einer Aenderung der Erregbarkeit gewisser Gehirncentren suchen zu müssen glaubt.

Die Kindermortalität stellt sich bei uns ebenfalls verhältnissmässig günstig. Von 490 Früchten kommen 9 als Aborte in Abzug. Von den restirenden 481 sind 333 lebend entlassen, 1 lebend zu Hause gelassen, 36 nach der Geburt gestorben, Summa $370=76,9$ pCt. der Gesammtzahl warden lebend geboren, so dass der Verlust vor und während der Geburt $23,1 \mathrm{pCt}$. beträgt, noch weniger also als die günstigen Zàhlen von Schauta (24,3 pCt.) and von Pawpertow (24,7 pCt.). Fon den Todtgebornen sind noch eine ganze Menge in Folge von entbindenden Operationen Zange, Wendung; Extraction - asphyctisch geworden und konnten 
nicht wiederbelebt werden, oder sind lebend perforirt worden, so dass sie auch nicht uuf Rechnung der Eclampsie zu setzen sind. Die eben genannten Zahlen drücken aber doch nicht den Einfluss der Eclampsie auf das Kind aus, denn es müssen erst abgezogen werden alle die Fälle, in denen die Eclampsie erst nach der Geburt des Kindes einsetzte. Solcher Kinder haben wir 152, incl. 2 Aborte, von denen 134 entlassen wurden und 7 erst nach der Geburt starben. Subtrahiren wir diese von den oben genannten 481 lebensfähigen Früchten, von denen 370 lebend geboren wurden, so erhalten wir 331 mit 229 lebend Geborenen, oder 69,1 pCt., während der Verlust 30,9 pCt. beträgt. Aber auch hier ist zu berücksichtigen, dass die grosse Zahl von Frühgeburten und von geburtshülflichen Operationen auch abgesehen von der Eclampsie die Sterblichkeit der Kinder wesentlich erhöhen muss.

Sowohl für die Mütter als für die Kinder haben wir an unserer Anstalt verhältnissmässig sehr günstige Resultate zu verzeichnen. Fragen wir daher jetzt nach der Therapie der Eclampsie, wie sie bei uns geübt worden ist. In den 20 Jahren, in denen unsere Fälle beobachtet worden sind, hat die Therapie der Eclampsie sich fast eben so wenig geändert, wie die Anschauungen über die Natur der Krankheit, war ziemlich rein empirisch und nahm nur bald mehr, bald weniger Rücksicht auf das eine oder das andere hervorstechende Symptom in dem Krankheitsbilde.

Da man die Eclampsie fast ausschliesslich erst zur Behandlung bekommt, wenn die Anfälle bereits ausgebrochen sind, so ist die Unterdrückung oder wenigstens die Milderung der letzteren meistentheils die erste Aufgabe, der man sich gegenübergestellt sieht. Da ferner, wie ich vor. Jahren experimentell nachgewiesen zu haben glaube (cf. Bidder, Holst's Beiträge II.), die nächste Ursache des Anfalles in einer acuten Ernährungslosigkeit des Gehirns oder wenigstens gewisser Theile desselben zu suchen ist, so sind, wie übrigens überall, bei uns die narkotischen Mittel bevorzugt und seit jeher energisch angewandt worden, unter ihnen besonders die Opiate, Opiumtinctur per anum and Morphium subcutan. Wir haben von denselben immer gute Erfolge gesehen, so dass die neuerliche, energische Empfehlung derselben durch G. Veit uns keineswegs überraschte. Stammt doch die Empfehlung grosser Opiumdosen schon aus dem vorigen Jahrhundert (Bland, 
Observat. on parturit. London, 1794) and brauche ich aus diesem Jahrhundert nur Kilian und Wiegand zu nennen ${ }^{1}$ ). Sicher spielt, neben der durch die Opiate hervorgerufenen Gehirnhyperämie, die gleichzeitig erreichte Herabsetzung der Reflexe eine nicht unwichtige Rolle.

Neben den Opiaten haben wir in demselben Sinne Chloroform und Chloralhydrat gebraucht, die Patienten öfters viele Stunden hindurch ohne Schaden in Chloroformnarcose erhalten; wir haben aber doch den Eindruck gewonnen, dass diese Mittel allein für sich nicht so viel wirken, als die Opiate. Letztere werden daher bei uns vorgezogen.

Neben den Anfällen nehmen aber die Oedeme, die Harnretention, die Nierenerkrankung in dem Krankheitsbilde der Eclampsie einen hervorragenden Platz ein. Es ist daher natürlich, dass der Steigerung der Diaphorese ein gebührender Raum in der Therapie angewiesen werden muss. So wird denn auch von Priessnitz'schen Einwickelungen und von heissen Bädern bei uns ein ausgiebiger Gebrauch gemacht.

Bei den bisher besprochenen Indicationen, der Hervorrufung von Gehirnhyperämie und der Steigerung der Diaphorese schien die Jaborandi, resp. das Pilocarpin zu genügen. Fehling (Centralblatt f. Gyn. 1878, No. 9) versuchte das erstere, ich (Centralblatt 1878, No. 15) das letztere. Das Resultat war, was die Unterdrückung der Anfälle betraf, ein ganz vorzügliches und bestätigte durchaus die Richtigkeit des theoretischen Raisonnements. Leider stellte es sich bald heraus, dass die durch Pilocarpin herrorgerufene Herzschwäche Eclamptischen gar zu leicht verderblich wird. Das sonst vortreffliche Mittel musste als zu gefährlich verlassen werden.

Alle anderen medicamentösen Mittel, die Nervina, die Diuretica, die Benzoesäure (gegen die Frerichs'sche Ammoniämie) u. s. w. haben meiner Meinung nach bei der Eclampsie gar keinen Werth.

Gehen wir zu der speciell geburtshülflichen Therapie über, so habe ich schon oben auseinandergesetzt, dass in etwa zwei Dritteln sämmtlicher Fälle von Eclampsia inter partum die Anfälle

1) cf. J. C. Schwartz. Ueber Eclampsie der Kreissenden. Aus den Rigaschen Beiträgen zur Heilkunde 1851. 
nach der Geburt des Kindes sofort aufhörten oder nur noch ein oder ein Paar Male in sehr gemilderter Form wiederkehrten, wir also viel Aussicht haben, die Eclampsie zu unterbrechen, wenn wir die Geburt des Kindes herbeiführen können. Dass diese Ueberlegung zu einer mehr activen Leitung der Geburt hindrängt, liegt auf der Hand, und so zählen wir denn unter unserem Material 169 Zangen, 6 Wendungen mit Extraction, 22 Extractionen bei Beckenendlage, 15 Expressionen nach Kristeller, 7 Perforationen mit Cranioclasie, 4 Kaiserschnitte; 3 künstliche Frühgeburten, abgesehen von den kleineren Operationen und Handgriffen. Nun sind freilich nicht alle diese Operationen ausgeführt worden im Sinne therapeutischer Maassregeln gegen die Eclampsie; einer ganzen Anzahl derselben haben die gewöhnlichen lndicationen zu Grunde gelegen, und die Eclampsie, die entweder später einsetzte, oder früher aufgehört hatte, oder so milde verlief, dass sie nicht in Betracht kam, hatte mit der Indicationsstellung nichts zu thun. Dennoch bleiben z. B. 66 Zangenoperationen, nach welchen die eclamptischen Anfälle wie abgeschnitten waren, und andere 40, nach welchen nur ein oder ein Paar schwächere und seltenere Anfälle zur Beobachtung kamen. Aehnliches sehen wir nach den anderen, sog. entbindenden Operationen. Wir haben also ganz bestimmt Recht, wenn wir überall da, wo die Bedingungen für relativ gefahrlose Entbindung gegeben sind, die Eclampsie an sich als Indication dafür ansehen, sogar die Bedingungen für die Entbindung in künstlicher Weise zu schaffen suchen. So ist es ganz bestimmt angezeigt, die Fruchtblase, die bei der Geburt im Wesentlichen nur eine hindernde Rolle spielt, möglichst früh zu sprengen, damit der Muttermundsrand sich direct über den vorliegenden Theil zurückziehen kann, was bei Eclampsie oft überraschend schnell geschieht. Ebenso bin ich einverstanden mit der energischen Art und Weise, in der Dührssen für tiefe Incisionen des Mutterhalses eingetreten ist, wenn die Erweiterung desselben sich verzögert. In der Ausdehnung, wie sie Dührssen verlangt, werden sie allerdings nur in den seltensten Fällen nöthig sein; denn, wenn der Muttermund so weit ist, oder um so viel erweitert wurde, dass die Zangenblätter hindurchgeführt werden können, so genügen meistentheils die Tractionen, um die Erweiterung zu vollenden.

Sehr zu beherzigen ist Dührssen's Rath, alle Operationen 
an Eclamptischen nur in tiefer Narcose vorzunehmen. Wir haben das seit jeher gethan und unsere günstigen Resultate mögen zum Theil auch dadurch bedingt sein.

Anders ist die Stellung, die ich gegenüber dem Kaiserschnitt, als entbindender Operation bei Eclampsie, einnehmen muss. Es war zu erwarten, dass, seit der Kaiserschnitt seine Schrecken verloren hat, er sein Gebiet immer weiter auszudehnen trachten würde. So hat er auch in's Gebiet der Eclampsie hinübergegriffen und Halbertsma beson ders hat schon eine Reihe von Fällen veröffentlicht, in denen er bei schwerer Eclampsie mit für Mutter und Kind günstigem Ausgange operirt hat. Trotz aller Verbesserung der Operationsmethoden und Vermeidung der Infection ist auch heute noch der Kaiserschnitt eine Operation, der einige Procente der Operirten zum Opfer fallen. Sind wir nun berechtigt, ein immerhin so gefährliches Mittel bei Eclampsie anzuwenden? Theoretisch muss darauf mit "Ja“ geantwortet werden, denn die Eclampsie ist noch viel gefährlicher. Wie wir oben gesehen haben, sterben von den Fällen, in denen 16 Anfälle und mehr beobachtet wurden, die wir also wohl als ,schwere" zu bezeichnen haben and die wohl allein bei der Indicationsstellung zum Kaiserschnitt in Betracht kommen dürften $-30-40 \mathrm{pCt}$. der Mütter. Wenn wir also sicher wüssten, dass die Entleerung des Uterus durch den Kaiserschnitt die Eclampsie beseitigt und wir es dann nur noch mit der Gefahr des Kaiserschnittes zu thun haben, so müssten wir bei Abwägung der Aussichten für die Kreissende den Kaiserschnitt vorziehen. Leider wissen wir das aber nicht. Wir sehen in einer grossen Zahl von Fällen die Anfälle, die während der Geburt eingesetzt hatten, auch nach der Entleerung des Uterus fortdauern, ja sogar an Heftigkeit zunehmen, oder aber der Sopor, das Lungenödem und die anderen Folgeerscheinungen der Anfälle führen, ohne dass weitere Anfälle auftreten, in mehr oder weniger langer Zeit nach der Geburt zum tödtlichen Ausgang. So etwas haben wir auch nach dem Kaiserschnitt gelegentlich zu erwarten und wir haben dann zu rechnen mit der Gefahr des Kaiserschnittes plus der Gefahr der Eclampsie. Die beiden Operationen, die wir an unserer Anstalt ausgeführt haben, hatten eben dieses Resultat, dass das fortdauernde Coma zum Tode führte.

Es ist ganz ohne Frage, dass das Bild, das eine eclamptische Kreissende schon nach dem ersten halben Dutzend von Anfällen 
darbietet, ein sehr schweres ist, so dass solche, die - wie Löhlein sich so effectroll ausdrückt - den heroischen Entscheidungen nach ihrem Temperament überhaupt den Vorzug vor dem Abwarten geben, schon hier sich gedrängt fühlen können, um jeden Preis Abhilfe zu schaffen. Dass man unter solchen Umständen leicht eine Reihe von Kaiserschnitten mit glücklichem Ausgange für Mutter und Kinder zusammenbringen könnte, liegt auf der Hand. Aber was wird dabei erreicht? An Eclampsie mit weniger als 16 Anfällen sterben, wie wir oben sahen, 5,5 pCt. der Mütter, am Kaiserschnitt -?

Man kann uns nicht verargen, dass wir bei dem günstigen Verlauf unserer Eclampsien uns zum Kaiserschnitt im Interesse der Mütter nur entschliessen könnten, wenn trotz aller sonstiger therapeutischer Maassregeln ,die Circulation und Respiration mehr und mehr gestört and beeinträchtigt erscheint und bei der im tiefen Sopor befindlichen Kranken Icterus auftritt" (Löhlein), vorausgesetzt natürlich, dass die Entbindung per vias naturales dabei unmöglich, resp. der Cervix noch nicht verstrichen ist. Unter solchen Umständen ist die zweite, von allen Autoren festgehaltene Bedingung, dass nämlich das Kind noch lebt, aber schwerlich jemals noch erfüllt.

Wenn man dagegen die Erfahrung hält, dass bei keiner Krankheit so häufig, wie bei der Eclampsie, das traurigste Krankheitsbild sich rasch ganz unerwarteter Weise freundlicher gestaltet, so dass im Einzelfall jede Prognose unmöglich wird, - (Löhlein erzählt einen schlagenden Fall, dem ich aus unserer reichen $\mathrm{Ca}-$ suistik so manchen ähnlichen an die Seite stellen könnte) - so bleibt für den Kaiserschnitt; als lebensrettende Operation bei Eclampsie, ein ganz ausserordentlich kleiner Raum übrig. Derselbe wird begrenzt einerseits durch das Aufgeben der Hoffnung auf jede anderweitige Therapie und andererseits durch den Beginn des Zustandes der Kreissenden, in dem wir sie als moribunda ansehen. Erreichen wir unter solchen Umständen durch die Operation nichts, so können wir uns nicht den Vorwurf machen, das letzte Mittel unversucht gelassen zu haben; ist das Resultat aber einmal günstig. so werden wir es als Gnadengeschenk dankbar registriren. Auf das Kind ist dabei nicht zu rechnen.

Ich habe an der Hand unseres Materiales in Kürze darzustellen versucht, was wir bisher im Kampfe mit der Eclampsie 
gewonnen haben und kann im Vergleich mit anderen Anstalten unsere Resultate als sehr günstige bezeichnen. Nichts desto weniger ist unsere Mortalität auch noch hoch genug. Möge durch die eben wieder lebhaft gewordene Arbeit auf dem Gebiete der Eclampsie unsere Erkenntniss des Wesens der Krankheit gefördert und uns neue Waffen geliefert werden, die besser, als die gegenwärtigen, uns in den Stand setzen, Mutter und Kind zu bewahren. 